\title{
Article \\ Estimating Ruin Probability in an Insurance Risk Model with Stochastic Premium Income Based on the CFS Method
}

\author{
Yujuan Huang ${ }^{1} \mathbb{D}$, Jing $\mathrm{Li}^{2}$, Hengyu Liu ${ }^{3}$ and Wenguang $\mathrm{Yu}^{4, *(\mathbb{D})}$ \\ 1 School of Science, Shandong Jiaotong University, Jinan 250357, China; 211018@sdjtu.edu.cn \\ 2 Department of Statistics and Actuarial Science, Chongqing University, Chongqing 401331, China; \\ li_jing@cqu.edu.cn \\ 3 Thurgood Marshall College, University of San Diego, San Diego, CA 92092, USA; hel107@ucsd.edu \\ 4 School of Insurance, Shandong University of Finance and Economics, Jinan 250014, China \\ * Correspondence: yuwg@sdufe.edu.cn
}

Citation: Huang, Y.; Li, J.; Liu, H.; Yu, W. Estimating Ruin Probability in an Insurance Risk Model with Stochastic Premium Income Based on the CFS Method. Mathematics 2021, 9, 982. https://doi.org/10.3390/math9090982

Academic Editor: Eric Ulm

Received: 24 March 2021

Accepted: 22 April 2021

Published: 27 April 2021

Publisher's Note: MDPI stays neutral with regard to jurisdictional claims in published maps and institutional affiliations.

Copyright: (c) 2021 by the authors. Licensee MDPI, Basel, Switzerland. This article is an open access article distributed under the terms and conditions of the Creative Commons Attribution (CC BY) license (https:/ / creativecommons.org/licenses/by/ $4.0 /)$.
Abstract: This paper considers the estimation of ruin probability in an insurance risk model with stochastic premium income. We first show that the ruin probability can be approximated by the complex Fourier series (CFS) expansion method. Then, we construct a nonparametric estimator of the ruin probability and analyze its convergence. Numerical examples are also provided to show the efficiency of our method when the sample size is finite.

Keywords: compound Poisson insurance risk model; stochastic processes; ruin probability; nonparametric estimation; complex Fourier series expansion

\section{Introduction}

In the classical insurance risk model, the premium rate is a constant and the premium collection is a linear function of time. Obviously, this assumption is not in line with the actual operation of the company. So, it is natural to extend the classical risk model by replacing the constant premium income with a compound Poisson process. For this purpose, this paper considers the following risk model that the premium income is no longer a linear function of time, but a stochastic process represented by a random sum, that is,

$$
U(t)=u+\sum_{i=1}^{M_{t}} Y_{i}-\sum_{i=1}^{N_{t}} X_{i}
$$

where, $u \geq 0$ is the initial surplus, the counting process of premium collection $M_{t}$ obeys the homogeneous Poisson process with parameter $\mu>0$, claim counting process $N_{t}$ obeys the homogeneous Poisson process with parameter $\lambda>0$. In addition, the amount of a single claim $X_{1}, X_{2}, \ldots$ is a series of continuous random variables which are independent and identically distributed in the random variable $X$, and the distribution function is $F_{X}$, the density function is $f_{X}$, the mean value is $\mu_{X}$. Similarly, the amount of a single premium $Y_{1}, Y_{2}, \ldots$ is a series of continuous random variables which are independent and identically distributed in the random variable $Y$, and the distribution function is $F_{Y}$, the density function is $f_{Y}$, the mean value is $\mu_{Y}$. In this model, we assume that $\left\{M_{t}\right\}_{t \geq 0},\left\{N_{t}\right\}_{t \geq 0},\left\{X_{i}\right\}_{i \geq 0},\left\{Y_{i}\right\}_{i \geq 0}$ are independent of each other, and the single premium income obeys the exponential distribution of parameter $\beta$, that is, the corresponding density function is $g(y)=\beta e^{-\beta y}, y>0$, and $\beta$ is unknown. Of course, we also need safe load conditions:

$$
\mu \cdot \mu_{Y}>\lambda \cdot \mu_{X}
$$


Define the ruin time $\tau=\inf \{t \geq 0: U(t)<0\}$, that is, the moment when $U(t)$ becomes negative for the first time. If $U(t) \geq 0$ for all $t \geq 0$, let $\tau=\infty$. When the initial capital $U(0)=u$ is given, we give the definition of the ruin probability:

$$
\psi(u)=P(\tau<\infty \mid U(0)=u), \quad u \geq 0 .
$$

Obviously, our model is more practical and more complex. We will continue to consider the approximation and estimation of ruin probability by using CFS expansion method in this model.

Ruin probability is the core content of risk theory, and its research has always been an enduring hot topic, related literature can be found Albrecher et al. [1], Mitric et al. [2], Li and Sendova [3], Bayraktar and Zhang [4], Li et al. [5], Mitric and Trufin [6], Li [7] and Han et al. [8]. Most of the above studies start from the convenience of model construction and solution, assuming that the company's surplus process satisfies a certain probability characteristic distribution, using Laplace transform, martingale, Hamilton-Jacobi-Bellman equation, dynamic programing principle and other methods to give the integro-differential equations satisfied by the relevant actuarial quantities or some specific expressions. In the actual business process of an insurance company, its surplus process, claim information, and other probability characteristics are unknown. Insurance companies only obtain a large amount of complex observational data through operations. Therefore, in recent years, some scholars have gradually begun to use statistical methods to study the calculation and nonparametric estimation of the relevant actuarial quantities of the classical risk model under the unknown probability characteristics. Zhang et al. [9] constructed the nonparametric estimation of the ruin probability for the classical risk model through the inverse Fourier transform and the kernel estimation method. Chau et al. [10] used the Fourier-Cosine series expansion method to estimate the uniform ruin probability and the Gerber-Shiu function. Goffard et al. [11] used the Laguerre method to study the nonparametric estimation of the ruin probability of the compound Poisson risk model. Yang et al. [12] estimated the severity of the ruin deficit through the Fourier-Cosine series expansion method. Wang et al. [13,14] estimated the Gerber-Shiu function and the expected discounted penalty function in a compound Poisson insurance risk model with stochastic premium income. Huang et al. [15] estimated the Gerber-Shiu expected discounted penalty function for Lévy risk model. For approximating the ruin probability and other risk measures, there are also some other methods. See for example Yu et al. [16], Zhang et al. [17], Su and Yu [18], Ai et al. [19], Wang et al. [20] and Cheung and Zhang [21].

Regarding the CFS expansion method, Chan [22] applied it to actuarial field for the first time, obtained the explicit pricing formula of European options, and proved the exponential convergence rate of this method. Chan [23] extended this method to the pricing calculation of price options with early movement characteristics under the index Lévy asset dynamics. The results show that this new method can quickly and accurately calculate early sports options. Through numerical analysis, the article also concludes that the CFS expansion method is computationally more comparable and superior than the current methods. We know that the current research on ruin theory, for example, the very popular Fourier-Cosine series expansion method is actually called real Fourier series methods. They decompose a given periodic function into $\sin (n x)$ and $\cos (n x)$ form. The CFS expansion method is another special alternative method, which uses complex numbers to decompose a given periodic function into the form of $e^{i n x}$. Li et al. [24] considered the nonparametric estimation of ruin probability by complex Fourier series expansion in the compound Poisson model. Wang et al. [20] studied the valuation problem of equity-linked annuity with guaranteed minimum death benefit by complex Fourier series method under regime-switching jump diffusion models.

In the ruin theory, the calculation, approximation and estimation of the probability of ruin has been a hot topic for a long time. The study of such problems provides effective early warning and technical support for the actual operation of insurance companies. Considering that the explicit formula of the probability of ruin can only be obtained under 
certain assumptions, in the face of more general situations, its explicit expression is usually not easy to obtain, and the approximation can only provide some rough information about ruin. In recent years, statistical estimation and inference have become an important means to deal with the problem of ruin probability. In recent years, a large number of studies use statistical methods of parametric and nonparametric estimation to study the ruin probability, and the sample data information used in them, such as the number of claims, the scale of individual claims, etc., are also obtained through observation in the actual operation of insurance companies. This method also gives the research certain feasibility and important practical significance. In this context, we hope to propose a nonparametric estimation method based on discrete observation data to estimate the ruin probability. At the same time, this method has good estimation properties, convergence speed and universal applicability. The general applicability here includes two aspects. On the one hand, we hope that the proposed estimation method can be applied to different risk models and different claim distribution assumptions. On the other hand, we hope that this estimation method can be extended to more risk models. If the above expectations can be met, it can be said that the research in this article is meaningful and valuable. It can not only provide corresponding theoretical guidance for different actual operating conditions, but also provide directions and references for further research by more scholars in the future.

In this paper, we shall use the CFS expansion method to estimate ruin probability in an insurance risk model with stochastic premium income. In fact, in most calculations, the expansion of CFS can make the problem simple and the formula refined, and only need to convert it to real Fourier series at the end. This paper applies this new method to the ruin field, and hopes that it can continue and enrich the research results in the field. The remainder of this paper is organized as follows. In Section 2, we introduce the CFS expansion method. In Section 3, we show how to approximate ruin probability by CFS expansion method. In Section 4, we give a nonparametric estimation of ruin probability. In Section 5, we study the approximation error caused by CFS approximation of ruin probability. In Section 6, some simulation results are given to show the effectiveness of our estimator. Finally, conclusions are given in Section 7.

\section{CFS Expansion Method}

In this section, we first give a basic introduction to the CFS expansion method. Define the function $f(x)$ on $[-\pi, \pi]$ to satisfy the following Dirichlet condition:

(1) $f(x)$ has a finite number of discontinuities on $[-\pi, \pi]$;

(2) $f(x)$ has a finite number of extreme values on $[-\pi, \pi]$;

(3) $\int_{-\pi}^{\pi}|f(x)| d x<\infty$.

Then, such a function $f(x)$ has the following CFS expansion expression:

$$
f(x)=\sum_{k=-\infty}^{\infty} A_{k} e^{i k x}
$$

where, the Fourier coefficients are given by

$$
A_{k}=\frac{1}{2 \pi} \int_{-\pi}^{\pi} f(x) e^{-i k x} d x, k=0, \pm 1, \pm 2, \ldots
$$

When faced with a more general situation, if the function $f(x)$ is defined on $[a, b]$ and satisfies the corresponding Dirichlet condition, through simple variable substitution, we similarly have:

$$
f(x)=\sum_{k=-\infty}^{\infty} A_{k} e^{i \frac{2 \pi}{b-a} k x},
$$


where

$$
A_{k}=\frac{1}{b-a} \int_{a}^{b} f(x) e^{-i \frac{2 \pi}{b-a} k x} d x, k=0, \pm 1, \pm 2, \ldots
$$

In practice, by introducing an appropriate truncated integer $K$, we can get an approximation of the function $f(x)$, namely:

$$
f(x) \approx f_{K}(x):=\sum_{k=-K}^{K} A_{k} e^{i \frac{2 \pi}{b-a} k x} .
$$

Since $f(x)$ is a real-valued function, take the real part of both sides of the equation at the same time, we get:

$$
f(x) \approx f_{K}(x):=\Re\left\{\sum_{k=-K}^{K} A_{k} e^{i \frac{2 \pi}{b-a} k x}\right\}=2 \sum_{k=0}^{K} \Re\left\{\left\{A_{k} e^{i \frac{2 \pi}{b-a} k x}\right\}\right.
$$

where, $\Re\{\cdot\}$ represents the real part of the formula, $\Sigma^{\prime}$ represents the summation form where the weight coefficient of the first summation item is $1 / 2$.

Equation (3) gives an approximation of the real-valued function that satisfies the Dirichlet condition. In the next section, we will use this conclusion to approximate and estimate the probability of ruin.

\section{Approximate Ruin Probability by CFS Expansion Method}

In this section, we will introduce how to apply the CFS method to approximate the probability of ruin.

First, we introduce the continuation of $\psi$ as follows:

$$
\psi_{\mathrm{e}}(u)=\left\{\begin{array}{rr}
\psi(u), & u \geq 0 \\
\psi(-u), & u<0 .
\end{array}\right.
$$

At the same time, define:

$$
\mathcal{F} \psi(s)=\int_{0}^{\infty} e^{i s u} \psi(u) d u, \mathcal{F} \psi_{e}(s)=\int_{-\infty}^{\infty} e^{i s u} \psi_{e}(u) d u, s \in \mathbb{R},
$$

respectively represent the Fourier transform of $\psi(u)$ and $\psi_{e}(u)$. On this basis, we have:

$$
\begin{aligned}
\mathcal{F} \psi_{e}(s) & =\int_{-\infty}^{\infty} e^{i s u} \psi_{e}(u) d u \\
& =\int_{0}^{\infty} e^{i s u} \psi(u) d u+\int_{-\infty}^{0} e^{i s u} \psi(-u) d u \\
& =\int_{0}^{\infty} e^{i s u} \psi(u) d u+\int_{0}^{\infty} e^{-i s u} \psi(u) d u \\
& =\mathcal{F} \psi(s)+\overline{\mathcal{F} \psi(s)}
\end{aligned}
$$

where, $\overline{\mathcal{F} \psi(s)}$ represents the conjugate complex number of $\mathcal{F} \psi(s)$.

Next, we study the process of using CFS method to approximate the ruin probability under the stochastic premium income insurance risk model. We define the function $\omega(u)=\int_{u}^{\infty} f_{X}(x) d x$, the Fourier transform and Laplace transform are respectively:

$$
\begin{gathered}
F \omega(s)=\int_{0}^{\infty} e^{i s u} \int_{u}^{\infty} f_{X}(x) d x d u=E\left(\int_{0}^{X} e^{i s u} d u\right), \mathrm{s} \in \mathbb{R}, \\
\mathcal{L} \omega(s)=\int_{0}^{\infty} e^{-s u} \int_{u}^{\infty} f_{X}(x) d x d u=E\left(\int_{0}^{X} e^{-s u} d u\right), \mathcal{R}(\mathrm{s}) \geq 0 .
\end{gathered}
$$

On the basis of these definitions, we first give the Fourier expression of $\psi_{e}(u)$.

From Equation (17) of Wang et al. [13], We know that the Fourier transform of $\psi(u)$ is:

$$
\mathcal{F} \psi(s)=\frac{\mathcal{F} H(s)}{1-\mathcal{F} G(s)}
$$


where,

$$
\begin{gathered}
\mathcal{F} H(s):=\frac{\lambda}{\lambda+\mu}\left[\mathcal{F} \omega(s)+\frac{\beta}{i s}\left(\mathcal{F} \omega(s)-\mu_{X}\right)\right], \\
\mathcal{F} G(s):=\frac{\lambda}{\lambda+\mu}\left[\mathcal{F} f(s)+\frac{\beta}{i s}(\mathcal{F} f(s)-1)\right] .
\end{gathered}
$$

By taking them into Equation (6), we get

$$
\mathcal{F} \psi_{e}(s)=\frac{\mathcal{F} H(s)}{1-\mathcal{F} G(s)}+\frac{\mathcal{F} H(-s)}{1-\mathcal{F} G(-s)} .
$$

Similarly, for the extended ruin probability $\psi_{e}(u)$, only considering that it is a function on $[-a, a]$, the CFS approximation of $\psi(u)$ can be obtained by using CFS approximation Equation (3):

$$
\psi(u) \approx \psi_{K}(u):=2 \sum_{k=0}^{K} \wedge \Re\left\{B_{k} e^{i \frac{k \pi}{a} u}\right\} I(0 \leq u \leq a),
$$

where, $I$ is an indicator function, and

$$
B_{k}=\frac{1}{2 a} \int_{-a}^{a} \psi_{e}(u) e^{-i \frac{k \pi}{a} u} d u, k=0,1, \ldots, K .
$$

Further, we have:

$$
B_{k} \approx \widetilde{B}_{k}:=\frac{1}{2 a} \int_{-\infty}^{\infty} \psi_{e}(u) e^{-i \frac{k \pi}{a} u} d u=\frac{1}{2 a} \mathcal{F} \psi_{e}\left(-\frac{k \pi}{a}\right),
$$

Finally, we get the approximate expression of ruin probability:

$$
\psi(u) \approx \widetilde{\psi}_{K}(u):=2 \sum_{k=0}^{K} \leadsto\left\{\widetilde{B}_{k} e^{i \frac{k \pi}{a} u}\right\} I(0 \leq u \leq a) .
$$

\section{Nonparametric Estimation of Ruin Probability}

In this section, we will give a nonparametric estimation of ruin probability based on the approximate results in the previous section.

It is assumed that insurance companies can obtain the following data sets:

(1) Data set composed of claim number and claim amount

$$
\left\{N_{T}, X_{1}, X_{2}, \ldots, X_{N_{T}}\right\},
$$

where $N_{T}$ is the total number of claims in the observation interval $[0, T]$.

(2) Data set composed of random premium income number and premium amount

$$
\left\{M_{T}, Y_{1}, Y_{2}, \ldots, Y_{M_{T}}\right\},
$$

where $M_{T}$ is the total number of random premium income in the observation interval $[0, T]$.

On this basis, we estimate the ruin probability. First, from Equations (7), (8), (10) and (11), we need to estimate the following parameters in order to construct the estimation of ruin probability:

$$
\lambda, \mu, \beta, \mathcal{F} \omega(s), \mathcal{F} f(s), \mu_{X}
$$

For $\lambda$ and $\mu$, we use the following estimators:

$$
\hat{\lambda}=\frac{1}{T} N_{T}, \hat{\mu}=\frac{1}{T} M_{T}
$$


Since the premium income $Y$ follows the exponential distribution with parameter $\beta$, then $E[Y]=1 / \beta$. From the moment estimation of expectation, we get the estimator of $\beta$ as follows:

$$
\hat{\beta}=\frac{1}{\frac{1}{M_{T}} \sum_{i=1}^{M_{T}} Y_{i}} .
$$

It is easily seen that:

$$
\hat{\lambda}-\lambda=O_{p}\left(T^{-\frac{1}{2}}\right), \hat{\mu}-\mu=O_{p}\left(T^{-\frac{1}{2}}\right), \hat{\beta}-\beta=O_{p}\left(T^{-\frac{1}{2}}\right) .
$$

Similarly, we can also use the moment estimator of $\mu_{X}$ to construct the corresponding estimator:

$$
\hat{\mu}_{X}=\frac{1}{N_{T}} \sum_{j=1}^{N_{T}} X_{j}
$$

For $\mathcal{F} \omega(s), \mathcal{F} f(s)$, according to the empirical characteristic function, we can get their estimators, respectively:

$$
\hat{\mathcal{F}} f(s)=\frac{1}{N_{T}} \sum_{j=1}^{N_{T}} e^{i s X_{j}}, \hat{\mathcal{F}} \omega(s)=\frac{1}{N_{T}} \sum_{j=1}^{N_{T}} \frac{e^{i S X_{j}}-1}{i s} .
$$

On this basis, according to Equations (7) and (8), we get the estimator of Fourier transform $\mathcal{F} \psi_{e}$ as follows:

$$
\hat{\mathcal{F}} \psi_{e}(s)=\frac{\hat{\mathcal{F}} H(s)}{1-\hat{\mathcal{F}} G(s)}+\frac{\hat{\mathcal{F} H}(-s)}{1-\hat{\mathcal{F} G}(-s)},
$$

where,

$$
\begin{gathered}
\hat{\mathcal{F} H}(s):=\frac{\hat{\lambda}}{\hat{\lambda}+\hat{\mu}}\left[\hat{\mathcal{F}} \omega(s)+\frac{\hat{\beta}}{i s}\left(\hat{\mathcal{F}} \omega(s)-\hat{\mu}_{x}\right)\right], \\
\hat{\mathcal{F}} G(s):=\frac{\hat{\lambda}}{\hat{\lambda}+\hat{\mu}}\left[\hat{\mathcal{F}} f(s)+\frac{\hat{\beta}}{i s}(\hat{\mathcal{F}} f(s)-1)\right] .
\end{gathered}
$$

Furthermore, combining with the Equations (10) and (11), we get the estimator expression of ruin probability:

$$
\hat{\psi}_{K}(u):=2 \sum_{k=0}^{K} \Re\left\{\left\{\hat{B}_{k} e^{i \frac{k \pi}{a} u}\right\} I(0 \leq u \leq a),\right.
$$

where,

$$
\hat{B}_{k}=\frac{1}{2 a} \hat{\mathcal{F}} \psi_{e}\left(-\frac{k \pi}{a}\right), k=0,1, \ldots, K .
$$

Obviously, the estimator expression we get is as concise as that of other models, which becomes a highlight of this estimation method.

\section{Convergence Analysis of Estimation Methods}

In this section, we will analyze the calculation error of the method proposed in the previous section. First, we study the approximation error caused by CFS approximation of ruin probability. The following proposition gives the corresponding conclusion.

Proposition 1. Suppose $\psi(u)$ is second order continuous differentiable, and $a=O(K),\left|i s \mathcal{F} f_{X}(s)\right|<C$, then for a very large $K$, we have:

$$
\sup _{u \geq 0}\left|\widetilde{\psi}_{K}(u)-\psi(u)\right| \leq \frac{2}{a} \int_{a}^{\infty} \psi(u) d u+\frac{a}{3}\left(\left|\psi^{\prime}(a)\right|+\int_{a}^{\infty}\left|\psi^{\prime \prime}(u)\right| d u\right)+\frac{c_{0} a}{\pi^{2} K}+\psi(a),
$$

where $c_{0}$ is a constant defined in the process of proving the proposition, and $C$ is also a constant. 
Proof. From the Equation (3.6) of Li et al. [24], we have

$$
\sup _{\mathbf{u} \geq 0}\left|\widetilde{\psi}_{K}(u)-\psi(u)\right| \leq 2 \sum_{k=0}^{\infty}\left|\widetilde{B}_{k}-B_{k}\right|+2 \sum_{k=K+1}^{\infty}\left|\widetilde{B}_{k}\right|+\psi(a) .
$$

In order to further give the upper bound of the Equation (15), we first calculate the residual sum $\sum_{k=K+1}^{\infty}\left|\widetilde{B}_{k}\right|$. Similarly, we need to study the Fourier transform $\mathcal{F} \psi_{e}(s)$. If we take Equations (7) into (8), we have:

$$
\begin{gathered}
\mathcal{F} \psi_{e}(s)=\frac{\mathcal{F} H(s)}{1-\mathcal{F} G(s)}+\frac{\mathcal{F} H(-s)}{1-\mathcal{F} G(-s)} \\
=\frac{\frac{\lambda}{\lambda+\mu}\left[\mathcal{F} \omega(s)+\frac{\beta}{i s}\left(\mathcal{F} \omega(s)-\mu_{x}\right)\right]}{1-\frac{\lambda}{\lambda+\mu}\left[\mathcal{F} f(s)+\frac{\beta}{i s}(\mathcal{F} f(s)-1)\right]}+\frac{\frac{\lambda}{\lambda+\mu}\left[\mathcal{F} \omega(-s)-\frac{\beta}{i s}\left(\mathcal{F} \omega(-s)-\mu_{x}\right)\right]}{1-\frac{\lambda}{\lambda+\mu}\left[\mathcal{F} f(-s)-\frac{\beta}{i s}(\mathcal{F} f(-s)-1)\right]}
\end{gathered}
$$

Since

$$
\mathcal{F} \omega(s)=E \int_{0}^{X} e^{i s u} d u=\frac{\mathcal{F} f_{X}(s)-1}{i s},
$$

bring it into the Equation (16) and simplify it, there are:

$$
\mathcal{F} \psi_{e}(s)=\frac{\beta\left(\lambda \mu-\mu_{X} \beta \lambda^{2}\right)\left(2-\mathcal{F} f_{X}(s)-\mathcal{F} f_{X}(-s)\right)+i s\left(\lambda \mu-\mu_{X} \beta \lambda^{2}\right)\left(\mathcal{F} f_{X}(-s)-\mathcal{F} f_{X}(s)\right)}{\left[\left(\lambda+\mu-\mathcal{F} f_{X}(s) \lambda\right) s+\beta \lambda i\left(\mathcal{F} f_{X}(s)-1\right)\right]\left[\left(\lambda+\mu-\mathcal{F} f_{X}(-s) \lambda\right) s+\beta \lambda i\left(1-\mathcal{F} f_{X}(-s)\right)\right]} .
$$

Due to

$$
\left|2-\mathcal{F} f_{X}(s)-\mathcal{F} f_{X}(-s)\right| \leq 4,\left|i s \mathcal{F} f_{X}(s)\right|<C,\left|i s \mathcal{F} f_{X}(-s)\right|<C,
$$

thus, we have

$$
\begin{gathered}
\left|\beta\left(\lambda \mu-\mu_{X} \beta \lambda^{2}\right)\left(2-\mathcal{F} f_{X}(s)-\mathcal{F} f_{X}(-s)\right)+i s\left(\lambda \mu-\mu_{X} \beta \lambda^{2}\right)\left(\mathcal{F} f_{X}(-s)-\mathcal{F} f_{X}(s)\right)\right| \\
<(4 \beta+2 C)\left(\lambda \mu+\mu_{X} \beta \lambda^{2}\right) .
\end{gathered}
$$

Similarly, when $|s|>2 \beta \lambda / \mu$, for the denominator, there is:

$$
\begin{gathered}
\mid\left[\left(\lambda+\mu-\mathcal{F} f_{X}(s) \lambda\right) s+\beta \lambda i\left(\mathcal{F} f_{X}(s)-1\right)\right]\left[\left(\lambda+\mu-\mathcal{F} f_{X}(-s) \lambda\right) s\right. \\
\left.+\beta \lambda i\left(1-\mathcal{F} f_{X}(-s)\right)\right] \mid \\
\geq\left(\left|\left(\lambda+\mu-\mathcal{F} f_{X}(s) \lambda\right) s\right|-\beta \lambda\left|\left(\mathcal{F} f_{X}(s)-1\right)\right|\right)\left(\left|\left(\lambda+\mu-\mathcal{F} f_{X}(-s) \lambda\right) s\right|\right. \\
\left.-\beta \lambda\left|\left(1-\mathcal{F} f_{X}(-s)\right)\right|\right) \geq(\mu|s|-2 \beta \lambda)^{2} .
\end{gathered}
$$

Thus, when $|s|>2 \beta \lambda / \mu$, we can get:

$$
\left|\mathcal{F} \psi_{e}(s)\right| \leq \frac{(4 \beta+2 C)\left(\lambda \mu+\mu_{X} \beta \lambda^{2}\right)}{(\mu|s|-2 \beta \lambda)^{2}} .
$$

In other words, when $|s| \rightarrow \infty$, there is $\left|\mathcal{F} \psi_{e}(s)\right|=O|s|^{-2}$, or in other words, there is a certain constant $c_{0}>0$, so that for a sufficiently large $|s|$, there is:

$$
\left|\mathcal{F} \psi_{e}(s)\right| \leq c_{0}|s|^{-2} \text {. }
$$

Thus, the coefficient $\widetilde{B}_{k}$ can be scaled to

$$
\left|\widetilde{B}_{k}\right| \leq \frac{1}{2 a}\left|\mathcal{F} \psi_{e}\left(-\frac{k \pi}{a}\right)\right| \leq \frac{c_{0} a}{2 k^{2} \pi^{2}} .
$$


On this basis, when $a=O(K)$, we can immediately get:

$$
\sum_{k=K+1}^{\infty}\left|\widetilde{B}_{k}\right| \leq \frac{c_{0} a}{2 \pi^{2}} \sum_{k=K+1}^{\infty} \frac{1}{k^{2}} \leq \frac{c_{0} a}{2 \pi^{2}} \sum_{k=K+1}^{\infty} \frac{1}{k(k-1)}=\frac{c_{0} a}{2 \pi^{2} K} .
$$

For the first formula on the right side of the inequality in Equation (15), Equation (3.14) of Li et al. [24] has already given the corresponding scaling result:

$$
2 \sum_{k=0}^{\infty}\left|\widetilde{B}_{k}-B_{k}\right| \leq \frac{2}{a} \int_{a}^{\infty} \psi(u) d u+\frac{a}{3}\left(\left|\psi^{\prime}(a)\right|+\int_{a}^{\infty}\left|\psi^{\prime \prime}(u)\right| d u\right) .
$$

Thus, integrating the Equations (15), (17) and (18), we finally get:

$$
\begin{aligned}
\sup _{\mathrm{u} \geq 0}\left|\widetilde{\psi}_{K}(u)-\psi(u)\right| \leq 2 \sum_{k=0}^{\infty}\left|\widetilde{B}_{k}-B_{k}\right|+2 \sum_{k=K+1}^{\infty}\left|\widetilde{B}_{k}\right|+\psi(a) \\
\leq \frac{2}{a} \int_{a}^{\infty} \psi(u) d u+\frac{a}{3}\left(\left|\psi^{\prime}(a)\right|+\int_{a}^{\infty}\left|\psi^{\prime \prime}(u)\right| d u\right)+\frac{c_{0} a}{\pi^{2} K}+\psi(a) .
\end{aligned}
$$

Note: The assumption $\mid$ is $\mathcal{F} f_{X}(s) \mid<C$ is reasonable for commonly used distributions, such as:

(1) When $X$ follows an exponential distribution with a parameter of $\alpha(\alpha>0)$, there is $\left|i s \mathcal{F} f_{X}(s)\right|=\left|\frac{i s \alpha}{\alpha-i s}\right|=\frac{\alpha}{\sqrt{\alpha^{2}+s^{2}}}|s| \stackrel{|s| \rightarrow \infty}{\longrightarrow} \alpha$, and thus $\left|i s \mathcal{F} f_{X}(s)\right|<C$.

(2) When $X$ obeys the Erlang distribution with parameter $(k, \lambda)\left(k \in \mathbb{N}_{+}, \lambda>0\right)$, there is $\left|i s \mathcal{F} f_{X}(s)\right|=\left|\frac{i s}{(1-i s / \lambda)^{k}}\right|=\left|\frac{i \lambda^{k}(\lambda+i s)^{k}}{\left(\lambda^{2}+s^{2}\right)^{k}}\right| \stackrel{|s| \rightarrow \infty}{\longrightarrow} 0, k \geq 2$, or $\lambda, k=1$. Thus we get $\left|i s \mathcal{F} f_{X}(s)\right|<C$.

Next, we study statistical errors. Two theorems are proposed here, and the conclusion will help us get the final proof result.

Theorem 1. Assuming $E X^{k}<\infty, k \in \mathbb{Z}$, and $a=O(K),\left\|H_{1}(x)\right\|_{P, 1}<\infty,\left\|H_{j}(x)\right\|_{P, 2}<\infty$, $j=1,2$, then for sufficiently large $K$ and $T$, we have:

$$
\sup _{s \in \mathcal{K}}|\hat{\mathcal{F} H}(s)-\mathcal{F} H(s)|=O_{p}\left(\frac{\sqrt{\log (K / a)}}{T}\right)
$$

where, $\mathcal{K}=\left\{\frac{k \pi}{a}: k=0, \pm 1, \ldots, \pm(K+1)\right\}$.

Proof. From the expressions of $\hat{\mathcal{F H}}(s)$ and $\mathcal{F} H(s)$, we know:

$$
\begin{aligned}
\hat{\mathcal{F}} H(s)-\mathcal{F} H(s)=\frac{\hat{\lambda}}{\hat{\lambda}+\hat{\mu}} \hat{\mathcal{F}} \omega(s) & -\frac{\lambda}{\lambda+\mu} \mathcal{F} \omega(s)+\frac{\hat{\lambda}}{\hat{\lambda}+\hat{\mu}} \hat{\frac{\beta}{i s}}\left(\hat{\mathcal{F}} \omega(s)-\hat{\mu}_{x}\right) \\
& -\frac{\lambda}{\lambda+\mu} \frac{\beta}{i s}\left(\mathcal{F} \omega(s)-\mu_{x}\right):=L_{1}+L_{2} .
\end{aligned}
$$

For $L_{1}$, we have

$$
\begin{gathered}
L_{1}=\frac{\hat{\lambda}}{\hat{\lambda}+\hat{\mu}} \hat{\mathcal{F}} \omega(s)-\frac{\lambda}{\lambda+\mu} \mathcal{F} \omega(s) \\
=\frac{\hat{\lambda}}{\hat{\lambda}+\hat{\mu}} \frac{1}{N_{T}} \sum_{j=1}^{N_{T}}\left(\int_{0}^{X_{j}} e^{i s u} d u-E \int_{0}^{X} e^{i s u} d u\right)+\left(\frac{\hat{\lambda}}{\hat{\lambda}+\hat{\mu}}-\frac{\lambda}{\lambda+\mu}\right) E \int_{0}^{X} e^{i s u} d u .
\end{gathered}
$$

Let $g_{1, s}(x)=\int_{0}^{x} e^{i s u} d u=\frac{e^{i s x}-1}{i s}$, then $L_{1}$ can be further expressed as

$$
L_{1}=\frac{\hat{\lambda}}{\hat{\lambda}+\hat{\mu}} \frac{1}{N_{T}} \sum_{j=1}^{N_{T}}\left(g_{1, s}\left(X_{j}\right)-E g_{1, s}(X)\right)+\left(\frac{\hat{\lambda}}{\hat{\lambda}+\hat{\mu}}-\frac{\lambda}{\lambda+\mu}\right) E g_{1, s}(X) .
$$


Since

$$
\sup _{\mathrm{s} \in \mathcal{K}}\left|E g_{1, \mathrm{~s}}(X)\right|=\sup _{\mathrm{s} \in \mathcal{K}}\left|E \int_{0}^{X} e^{i s u} d u\right| \leq \sup _{\mathrm{s} \in \mathcal{K}}|E X|=|E X|<\infty,
$$

then according to $\hat{\lambda}-\lambda=O_{p}\left(T^{-\frac{1}{2}}\right), \hat{\mu}-\mu=O_{p}\left(T^{-\frac{1}{2}}\right)$, the second formula on the right side of Equation (20) can be further proved as:

$$
\sup _{\mathbf{s} \in \mathcal{K}}\left|\left(\frac{\hat{\lambda}}{\hat{\lambda}+\hat{\mu}}-\frac{\lambda}{\lambda+\mu}\right) E g_{1, s}(X)\right| \leq\left|\frac{\hat{\lambda}}{\hat{\lambda}+\hat{\mu}}-\frac{\lambda}{\lambda+\mu}\right| \sup _{\mathbf{s} \in \mathcal{K}}\left|\operatorname{Eg} g_{1, s}(X)\right|=O_{p}\left(T^{-\frac{1}{2}}\right) .
$$

In addition, from the Lemma 3.1 of Li et al. [24], the first formula on the right side of Equation (20) can be further processed as:

$$
\begin{aligned}
\sup _{s \in \mathcal{K}}\left|\frac{\hat{\lambda}}{\hat{\lambda}+\hat{\mu}} \frac{1}{N_{T}} \sum_{j=1}^{N_{T}}\left(g_{1, s}\left(X_{j}\right)-E g_{1, s}(X)\right)\right|=\sup _{s \in \mathcal{K}}\left|\frac{\hat{\lambda}}{\hat{\lambda}+\hat{\mu}} \frac{1}{N_{T}} \sum_{j=1}^{N_{T}}\left(\frac{e^{i s X_{j}}-1}{i s}-E \frac{e^{i s X}-1}{i s}\right)\right| \\
=\sup _{s \in \mathcal{K}}\left|\frac{\hat{\lambda}}{\hat{\lambda}+\hat{\mu}} \frac{1}{N_{T}} \sum_{j=1}^{N_{T}}\left(\frac{e^{i s X_{j}}-1}{i s}-E \frac{e^{i s X}-1}{i s}\right)\right|=\sup _{s \in \mathcal{K}}\left|\frac{\hat{\lambda}}{\hat{\lambda}+\hat{\mu}} \frac{1}{N_{T}} \sum_{j=1}^{N_{T}}\left(\frac{e^{i s X_{j}}-E e^{i s X}}{i s}\right)\right| \\
=\sup _{s \in \mathcal{K}}\left|\frac{\hat{\lambda}}{\hat{\lambda}+\hat{\mu}} \frac{1}{N_{T}} \sum_{j=1}^{N_{T}}\left(\frac{e^{i s X_{j}}-E e^{i s X}}{i s}\right)\right|=\sup _{s \in \mathcal{K}}\left|\frac{\hat{\lambda}}{\hat{\lambda}+\hat{\mu}} \frac{\mathcal{F} f_{X}(s)-\hat{\mathcal{F}} f_{X}(s)}{i s}\right| \\
=\sup _{s \in \mathcal{K}}\left|\frac{\hat{\lambda}}{\hat{\lambda}+\hat{\mu}} \frac{\mathcal{F} f_{X}(s)-\hat{\mathcal{F}} f_{X}(s)}{i s}\right|=O_{p}\left(\frac{\sqrt{\log (K / a)}}{T}\right) .
\end{aligned}
$$

Combining Equation (21), we get:

$$
\sup _{\mathrm{s} \in \mathcal{K}}\left|L_{1}\right|=O_{p}\left(\frac{\sqrt{\log \left(\frac{K}{a}\right)}}{T}\right) .
$$

Next consider $L_{2}$. Bringing the specific expressions of $\hat{\mathcal{F} \omega}(s), \hat{\mu}_{x}$ and $\mathcal{F} \omega(s)$ into $L_{2}$, we get the following formula:

$$
L_{2}=\frac{\hat{\lambda} \hat{\beta}}{\hat{\lambda}+\hat{\mu}} \frac{1}{N_{T}} \sum_{j=1}^{N_{T}} \frac{\int_{0}^{X_{j}} e^{i s u} d u-X_{j}}{i s}-\frac{\lambda \beta}{\lambda+\mu} E\left(\frac{\int_{0}^{X} e^{i s u} d u-X}{i s}\right) .
$$

Let $g_{2, s}(x)=\frac{\int_{0}^{x} e^{i s u} d u-x}{i s}$, then

$$
\begin{aligned}
L_{2} & =\frac{\hat{\lambda} \hat{\beta}}{\hat{\lambda}+\hat{\mu}} \frac{1}{N_{T}} \sum_{j=1}^{N_{T}} g_{2, s}\left(X_{j}\right)-\frac{\lambda \beta}{\lambda+\mu} E g_{2, s}(X) \\
& =\frac{\hat{\lambda} \hat{\beta}}{\hat{\lambda}+\hat{\mu}} \frac{1}{N_{T}} \sum_{j=1}^{N_{T}}\left(g_{2, s}\left(X_{j}\right)-E g_{2, s}(X)\right)+\left(\frac{\hat{\lambda} \hat{\beta}}{\hat{\lambda}+\hat{\mu}}-\frac{\lambda \beta}{\lambda+\mu}\right) E g_{2, s}(X) .
\end{aligned}
$$

For the second equation on the right side of the last equation, considering:

$$
\begin{aligned}
g_{2, s}(X) & =\frac{\int_{0}^{X} e^{i s u} d u-X}{i s}=\int_{0}^{X} \frac{e^{i s u}-1}{i s} d u \\
& =\int_{0}^{X} \int_{0}^{u} e^{i s(u-y)} d y d u \leq \int_{0}^{X} \int_{0}^{u} d y d u=\frac{1}{2} X^{2},
\end{aligned}
$$

then combined with $\hat{\lambda}-\lambda=O_{p}\left(T^{-\frac{1}{2}}\right), \hat{\mu}-\mu=O_{p}\left(T^{-\frac{1}{2}}\right), \hat{\beta}-\beta=O_{p}\left(T^{-\frac{1}{2}}\right)$, we know:

$$
\sup _{\mathrm{s} \in \mathcal{K}}\left|\left(\frac{\hat{\lambda} \hat{\beta}}{\hat{\lambda}+\hat{\mu}}-\frac{\lambda \beta}{\lambda+\mu}\right) E g_{2, s}(X)\right| \leq \frac{1}{2} \sup _{\mathrm{s} \in \mathcal{K}}\left|\frac{\hat{\lambda} \hat{\beta}}{\hat{\lambda}+\hat{\mu}}-\frac{\lambda \beta}{\lambda+\mu}\right|\left|E X^{2}\right|=O_{p}\left(T^{-\frac{1}{2}}\right) .
$$


At the same time, for the first formula on the right side of the last equation of Equation (24), we need to refer to the proof ideas in the appendix of Zhang [25], then we have:

$$
\sup _{\mathbf{s} \in \mathcal{K}}\left|\frac{1}{N_{T}} \sum_{j=1}^{N_{T}}\left(g_{2, s}\left(X_{j}\right)-E g_{2, s}(X)\right)\right|=O_{p}\left(\frac{\sqrt{\log (K / a)}}{T}\right),
$$

then

$$
\sup _{\mathbf{s} \in \mathcal{K}}\left|\frac{\hat{\lambda} \hat{\beta}}{\hat{\lambda}+\hat{\mu}} \frac{1}{N_{T}} \sum_{j=1}^{N_{T}}\left(g_{2, s}\left(X_{j}\right)-E g_{2, s}(X)\right)\right|=O_{p}\left(\frac{\sqrt{\log \left(\frac{K}{a}\right)}}{T}\right) .
$$

Combining Equations (25) and (26), we get:

$$
\sup _{\mathbf{s} \in \mathcal{K}}\left|L_{2}\right|=O_{p}\left(\frac{\sqrt{\log \left(\frac{K}{a}\right)}}{T}\right) .
$$

Finally, it is proved by Equations (19), (23) and (27).

Theorem 1 shows that the estimation error of $\mathcal{F} H(s)$ has logarithmic convergence. In order to further give the estimated error convergence rate of $\widetilde{\psi}_{K}(u)$, we need to analyze $\mathcal{F} G(s)$ next. The following theorem will solve our considerations well.

Theorem 2. Assuming $E X^{k}<\infty, k \in \mathbb{Z}$, for sufficiently large $a, K$ and $T$, we have:

$$
\sup _{s \in \mathcal{K}}|\hat{\mathcal{F}} G(s)-\mathcal{F} G(s)|=O_{p}\left(\frac{\sqrt{\log (K / a)}}{T}\right),
$$

where, $\mathcal{K}=\{k \pi / a: k=0, \pm 1, \ldots, \pm(K+1)\}$.

Proof. From the expressions of $\hat{\mathcal{F}} G(s)$ and $\mathcal{F} G(s)$, we know:

$$
\begin{aligned}
\hat{\mathcal{F} G}(s)-\mathcal{F} G(s)=\frac{\hat{\lambda}}{\hat{\lambda}+\hat{\mu}} \hat{\mathcal{F}} f(s) & -\frac{\lambda}{\lambda+\mu} \mathcal{F} f(s)+\frac{\hat{\lambda}}{\hat{\lambda}+\hat{\mu}} \hat{i}(\hat{\mathcal{F}} f(s)-1) \\
& -\frac{\lambda}{\lambda+\mu} \frac{\beta}{i s}(\mathcal{F} f(s)-1):=\Pi_{1}+\Pi_{2} .
\end{aligned}
$$

For $\Pi_{1}$, let $g_{3, s}(x)=e^{i s x}$, then further get:

$$
\begin{aligned}
\Pi_{1} & =\frac{\hat{\lambda}}{\hat{\lambda}+\hat{\mu}} \hat{\mathcal{F}} f(s)-\frac{\lambda}{\lambda+\mu} \mathcal{F} f(s) \\
& =\frac{\hat{\lambda}}{\hat{\lambda}+\hat{\mu}} \frac{1}{N_{T}} \sum_{j=1}^{N_{T}}\left(e^{i s X_{j}}-E e^{i s X}\right)+\left(\frac{\hat{\lambda}}{\hat{\lambda}+\hat{\mu}}-\frac{\lambda}{\lambda+\mu}\right) E e^{i s X}, \\
& =\frac{\hat{\lambda}}{\hat{\lambda}+\hat{\mu}} \frac{1}{N_{T}} \sum_{j=1}^{N_{T}}\left(g_{3, s}\left(X_{j}\right)-E g_{3, s}(X)\right)+\left(\frac{\hat{\lambda}}{\hat{\lambda}+\hat{\mu}}-\frac{\lambda}{\lambda+\mu}\right) E g_{3, s}(X) .
\end{aligned}
$$

Since

$$
\sup _{\mathbf{s} \in \mathcal{K}}\left|E g_{3, s}(X)\right| \leq 1<\infty, \hat{\lambda}-\lambda=O_{p}\left(T^{-\frac{1}{2}}\right), \hat{\mu}-\mu=O_{p}\left(T^{-\frac{1}{2}}\right),
$$

thus, for the second term on the right side of the last equation in Equation (29), we have:

$$
\sup _{\mathbf{s} \in \mathcal{K}}\left|\left(\frac{\hat{\lambda}}{\hat{\lambda}+\hat{\mu}}-\frac{\lambda}{\lambda+\mu}\right) E g_{3, s}(X)\right|=O_{p}\left(T^{-\frac{1}{2}}\right) .
$$


For the first term on the right side of the last equation in Equation (29), we consider introducing two real-valued function classes:

$$
\begin{aligned}
& g_{3, k, R}=\left\{g: g=\operatorname{Re}\left(g_{3, s}\right), s \in \mathcal{K}\right\}, \\
& g_{3, k, I}=\left\{g: g=\operatorname{Im}\left(g_{3, s}\right), s \in \mathcal{K}\right\} .
\end{aligned}
$$

Then

$$
\begin{gathered}
\sup _{\mathrm{s} \in \mathcal{K}}\left|\frac{1}{N_{T}} \sum_{j=1}^{N_{T}}\left(g_{3, s}\left(X_{j}\right)-E g_{3, s}(X)\right)\right| \\
\leq \sup _{g \in g_{3, k, R}}\left|\frac{1}{N_{T}} \sum_{j=1}^{N_{T}}\left(g\left(X_{j}\right)-E g(X)\right)\right|+\sup _{g \in g_{3, k, I}}\left|\frac{1}{N_{T}} \sum_{j=1}^{N_{T}}\left(g\left(X_{j}\right)-E g(X)\right)\right| .
\end{gathered}
$$

Refer to the proof of Wang et al. [13], we know:

$$
\begin{aligned}
& \sup _{g \in g_{3, k, R}}\left|\frac{1}{N_{T}} \sum_{j=1}^{N_{T}}\left(g\left(X_{j}\right)-E g(X)\right)\right|=O_{p}\left(\frac{\sqrt{\log (K / a)}}{T}\right), \\
& \sup _{g \in g_{3, k, I}}\left|\frac{1}{N_{T}} \sum_{j=1}^{N_{T}}\left(g\left(X_{j}\right)-E g(X)\right)\right|=O_{p}\left(\frac{\sqrt{\log (K / a)}}{T}\right) .
\end{aligned}
$$

Thus, we have

$$
\sup _{\mathbf{s} \in \mathcal{K}}\left|\frac{\hat{\lambda}}{\hat{\lambda}+\hat{\mu}} \frac{1}{N_{T}} \sum_{j=1}^{N_{T}}\left(g_{3, s}\left(X_{j}\right)-E g_{3, s}(X)\right)\right|=O_{p}\left(\frac{\sqrt{\log \left(\frac{K}{a}\right)}}{T}\right) .
$$

Combining Equations (29), (30) and (31), the maximum boundary of $\Pi_{1}$ can be obtained:

$$
\sup _{s \in \mathcal{K}}\left|\Pi_{1}\right|=O_{p}\left(\frac{\sqrt{\log \left(\frac{K}{a}\right)}}{T}\right) .
$$

Next consider $\Pi_{2}$. Let $g_{4, s}(x)=\frac{e^{i s x}-1}{i s}$, then:

$$
\begin{aligned}
\Pi_{2} & =\frac{\hat{\lambda}}{\hat{\lambda}+\hat{\mu}} \frac{\hat{\beta}}{i s}(\hat{\mathcal{F}} f(s)-1)-\frac{\lambda}{\lambda+\mu} \frac{\beta}{i s}(\mathcal{F} f(s)-1) \\
& =\frac{\hat{\lambda} \hat{\beta}}{\hat{\lambda}+\hat{\mu}} \frac{1}{N_{T}} \sum_{j=1}^{N_{T}} \frac{e^{i s X_{j}}-1}{i s}-\frac{\lambda \beta}{\lambda+\mu} E \frac{e^{i s} X_{-1}}{i s} \\
& =\frac{\hat{\lambda} \hat{\beta}}{\hat{\lambda}+\hat{\mu}} \frac{1}{N_{T}} \sum_{j=1}^{N_{T}}\left(g_{4, s}\left(X_{j}\right)-E g_{4, s}(X)\right)+\left(\frac{\hat{\lambda} \hat{\beta}}{\hat{\lambda}+\hat{\mu}}-\frac{\lambda \beta}{\lambda+\mu}\right) E g_{4, s}(X) .
\end{aligned}
$$

Since

$$
g_{4, S}(x)=\frac{e^{i s x}-1}{i s}=\int_{0}^{x} e^{i s(x-y)} d y \leq x,
$$

then combined with $\hat{\lambda}-\lambda=O_{p}\left(T^{-\frac{1}{2}}\right), \hat{\mu}-\mu=O_{p}\left(T^{-\frac{1}{2}}\right), \hat{\beta}-\beta=O_{p}\left(T^{-\frac{1}{2}}\right)$, we can get:

$$
\sup _{\mathrm{s} \in \mathcal{K}}\left|\left(\frac{\hat{\lambda} \hat{\beta}}{\hat{\lambda}+\hat{\mu}}-\frac{\lambda \beta}{\lambda+\mu}\right) E g_{4, s}(X)\right| \leq \sup _{\mathbf{s} \in \mathcal{K}}\left|\frac{\hat{\lambda} \hat{\beta}}{\hat{\lambda}+\hat{\mu}}-\frac{\lambda \beta}{\lambda+\mu}\right||E X|=O_{p}\left(T^{-\frac{1}{2}}\right) .
$$


Next consider the first equation on the right side of the last equation of Equation (33). Due to the Lemma 3.1 of Li et al. [24], then we have:

$$
\begin{gathered}
\sup _{\mathbf{s} \in \mathcal{K}}\left|\frac{\hat{\lambda} \hat{\beta}}{\hat{\lambda}+\hat{\mu}} \frac{1}{N_{T}} \sum_{j=1}^{N_{T}}\left(g_{4, s}\left(X_{j}\right)-E g_{4, s}(X)\right)\right| \\
=\frac{\hat{\lambda} \hat{\beta}}{\hat{\lambda}+\hat{\mu}} \sup _{\mathrm{s} \in \mathcal{K}}\left|\frac{1}{N_{T}} \sum_{j=1}^{N_{T}} \frac{e^{i s X_{j}}-E e^{i s X}}{i s}\right| \\
=\frac{\hat{\lambda} \hat{\beta}}{\hat{\lambda}+\hat{\mu}} \sup _{\mathbf{s} \in \mathcal{K}}\left|\frac{\mathcal{F} f_{X}(s)-\hat{\mathcal{F}} f_{X}(s)}{i s}\right|=O_{p}\left(\frac{\sqrt{\log \left(\frac{K}{a}\right)}}{T}\right) .
\end{gathered}
$$

Combining Equations (34) and (35), we get:

$$
\sup _{\mathrm{s} \in \mathcal{K}}\left|\Pi_{2}\right|=O_{p}\left(\frac{\sqrt{\log \left(\frac{K}{a}\right)}}{T}\right) .
$$

Finally, combining the conclusions of Equations (28), (32) and (36), the theorem is proved.

On the basis of the above two propositions, we propose the final estimation error conclusion of the ruin probability:

Proposition 2. Assume $E X^{k}<\infty, k \in \mathbb{Z}$ and $a=O(K),\left\|H_{1}(x)\right\|_{P, 1}<\infty,\left\|H_{j}(x)\right\|_{P, 2}<\infty$, $j=1,2$, then for very large $a, K$ and $T$, we have:

$$
\sup _{\mathbf{u} \geq 0}\left|\widetilde{\psi}_{K}(u)-\hat{\psi}_{K}(u)\right|=O_{p}\left((K / a) \frac{\sqrt{\log (K / a)}}{T}\right)
$$

Proof. According to the expressions of $\mathcal{F} \psi_{e}(s)$ and $\hat{\mathcal{F}} \psi_{e}(s)$, we know

$$
\begin{aligned}
& \sup _{\mathrm{s} \in \mathcal{K}}\left|\mathcal{F} \psi_{e}(s)-\hat{\mathcal{F}} \psi_{e}(s)\right| \\
& =\sup _{s \in \mathcal{K}}\left|\frac{\mathcal{F} H(s)}{1-\mathcal{F} G(s)}+\frac{\mathcal{F} H(-s)}{1-\mathcal{F} G(-s)}-\frac{\hat{\mathcal{F}} H(s)}{1-\mathcal{F}^{\hat{F}} G(s)}-\frac{\hat{\mathcal{F} H}(-s)}{1-\mathcal{F}^{\hat{F}} G(-s)}\right| \\
& \leq \sup _{s \in \mathcal{K}}\left|\frac{\mathcal{F} H(s)}{1-\mathcal{F} G(s)}-\frac{\hat{\mathcal{F}} H(s)}{1-\hat{\mathcal{F}}^{G} G(s)}\right|+\sup _{\mathrm{s} \in \mathcal{K}}\left|\frac{\mathcal{F} H(-s)}{1-\mathcal{F} G(-s)}-\frac{\hat{\mathcal{F} H}(-s)}{1-\hat{\mathcal{F}}^{\hat{G}} G(-s)}\right| \\
& =2 \sup _{s \in \mathcal{K}}\left|\frac{\mathcal{F} H(s)}{1-\mathcal{F} G(s)}-\frac{\hat{\mathcal{F} H}(s)}{1-\mathcal{F} G(s)}\right| \\
& =2 \sup _{s \in \mathcal{K}}\left|\frac{(1-\mathcal{F} G(s))(\mathcal{F} H(s)-\hat{\mathcal{F}} H(s))+\mathcal{F} H(s)(\mathcal{F} G(s)-\hat{\mathcal{F}} G(s))}{(1-\mathcal{F} G(s))(1-\hat{\mathcal{F}} G(s))}\right| .
\end{aligned}
$$

Because of $\left|\frac{1-\hat{\mathcal{F}} f(s)}{i s}\right| \leq \frac{1}{N_{T}} \sum_{j=1}^{N_{T}} X_{j},\left|\frac{1-\mathcal{F} f(s)}{i s}\right| \leq E X,|\hat{\mathcal{F}} f(s)| \leq 1,\left|\frac{\mathcal{F} \omega(s)-\mu_{x}}{i s}\right| \leq \frac{1}{2} E X^{2}$, a simple conclusion can be drawn:

$$
|1-\hat{\mathcal{F}} G(s)|=\left|1-\frac{\hat{\lambda}}{\hat{\lambda}+\hat{\mu}}\left[\begin{array}{c}
\hat{\mathcal{F}} f(s)+ \\
\frac{\hat{\beta}}{i s}(\hat{\mathcal{F}} f(s)-1)
\end{array}\right]\right|
$$




$$
\begin{gathered}
\geq 1-\frac{\hat{\lambda}}{\hat{\lambda}+\hat{\mu}}\left(|\hat{\mathcal{F}} f(s)|+\hat{\beta}\left|\frac{1-\hat{\mathcal{F}} f(s)}{i s}\right|\right) \\
\geq 1-\frac{\hat{\lambda}}{\hat{\lambda}+\hat{\mu}}\left(1+\hat{\beta} \frac{1}{N_{T}} \sum_{j=1}^{N_{T}} X_{j}\right)=C_{1}, \\
|1-\mathcal{F} G(s)|=\left|1-\frac{\lambda}{\lambda+\mu}\left[\mathcal{F} f(s)+\frac{\beta}{i s}(\mathcal{F} f(s)-1)\right]\right| \\
\geq 1-\frac{\lambda}{\lambda+\mu}\left(|\mathcal{F} f(s)|+\beta\left|\frac{1-\mathcal{F} f(s)}{i s}\right|\right) \\
\geq 1-\frac{\lambda}{\lambda+\mu}(1+\beta E X)=C_{2} .
\end{gathered}
$$

Incorporating the above conclusions into Equation (37), we get:

$$
\sup _{\mathrm{s} \in \mathcal{K}}\left|\mathcal{F} \psi_{e}(s)-\hat{\mathcal{F}} \psi_{e}(s)\right|=O_{p}\left(\frac{\sqrt{\log (K / a)}}{T}\right) .
$$

Combined with the Equation (3.15) of Li et al. [24], finally, we have:

$$
\begin{aligned}
\sup _{\mathrm{u} \geq 0}\left|\widetilde{\psi}_{K}(u)-\hat{\psi}_{K}(u)\right| \leq & \frac{K+1}{a} \sup _{\mathrm{s} \in \mathcal{K}}\left|\mathcal{F} \psi_{e}(s)-\hat{\mathcal{F}} \psi_{e}(s)\right| \\
\leq & \frac{K+1}{a} \sup _{\mathrm{s} \in \mathcal{K}}\left|\mathcal{F} \psi_{e}(s)-\hat{\mathcal{F}} \psi_{e}(s)\right| \\
& =O_{p}\left((K / a) \frac{\sqrt{\log (K / a)}}{T}\right) \cdot \square
\end{aligned}
$$

\section{Numerical Simulation}

In this section, we will provide some simulation results to show the estimation effect of our estimation method when the sample size is limited. We set the parameters $\lambda=2, \mu=5$, $\beta=1, K=1024$. In addition, let $a=30$, that is $0 \leq u \leq 30$. This is because when the initial surplus $u>30$, the value of ruin probability is very close to 0 , then we consider the following three claim density functions at the same time:

(1) Exponential density function: $f_{X}(x)=e^{-x}, x>0$.

(2) Erlang (2) density function: $f_{X}(x)=4 x e^{-2 x}, x>0$.

(3) Gamma $(1.5,1.5)$ density function: $f_{X}(x)=\frac{1.5^{1.5} x^{0.5} e^{-1.5 x}}{\Gamma(1.5)}, x>0$, where $\Gamma(\cdot)$ is the gamma function.

Note that the assumptions of the above three claim density functions all satisfy $\mu_{X}=1$, and through the Laplace transform method, the true value of the ruin probability under the first two claim distribution assumptions can be directly calculated, respectively:

(1) Exponential density function: $\psi(u)=0.5714 e^{-0.4286 u}, u \geq 0$.

(2) Erlang (2) density function: $\psi(u)=0.5714 e^{-1.8571 u}(\cosh (1.3171 u)+1.2474 \sinh (1.3171 u))$, $u \geq 0$.

For the third density hypothesis Gamma $(1.5,1.5)$ density function, we also compare the approximate simulation results with the estimated results. We will consider the case of $T=120,180,360$, and calculate the integral mean square error (IMSE), mean value and average relative error respectively based on 300 independent repeated experiments.

First of all, Figures 1-3 show the simulation effect of 25 independent repeated experiments under three kinds of claim distribution. At the same time, the corresponding effect is compared with the true value curve to illustrate the stability and accuracy of the program, and fully show the different variation bands of estimated values under different observation intervals. It can be clearly observed from the Figures that with the increase of observation time $T$, the estimation tends to be more and more stable. 


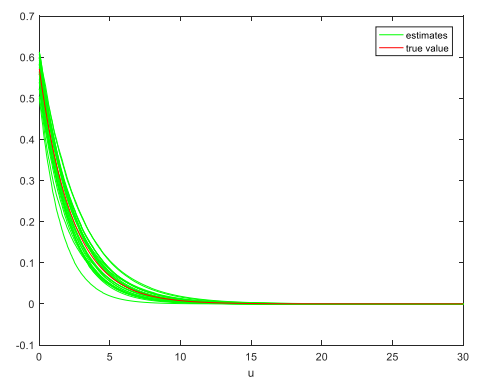

(a)

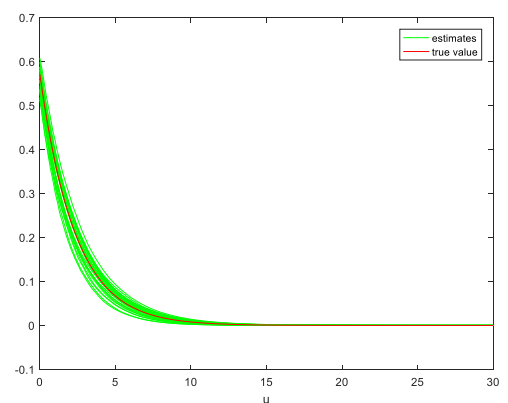

(b)

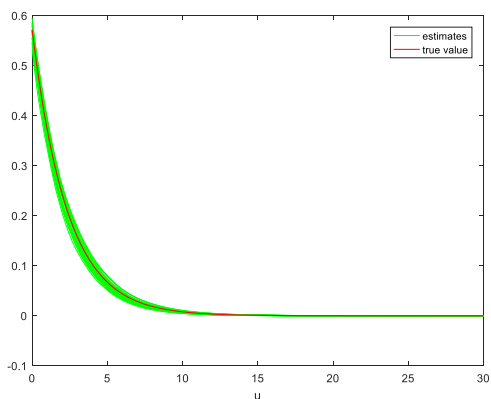

(c)

Figure 1. Estimation of the ruin probability for exponential claim size density: (a) $T=120$, (b) $T=180$, (c) $T=360$.

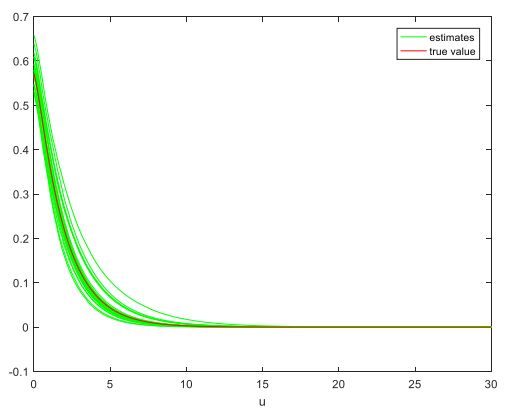

(a)

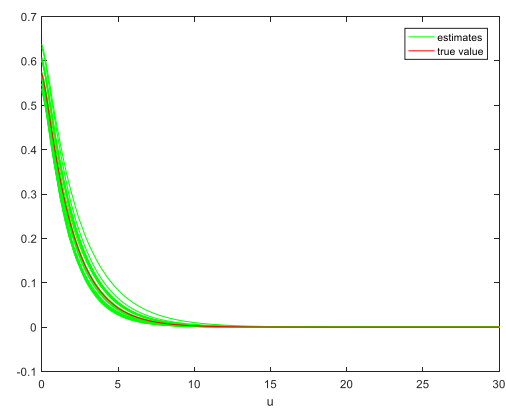

(b)

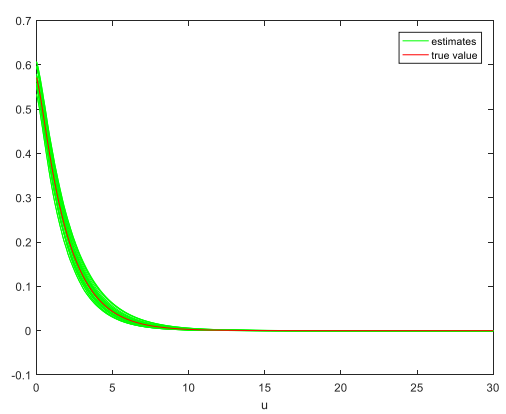

(c)

Figure 2. Estimation of the ruin probability for Erlang (2) claim size density: (a) $T=120,(\mathbf{b}) T=180$, (c) $T=360$.

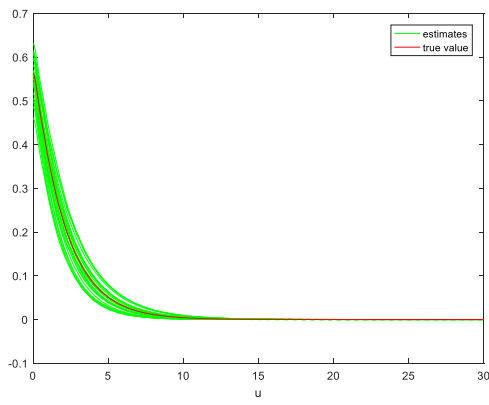

(a)

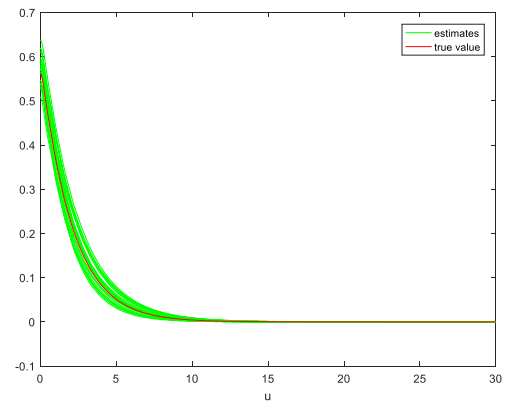

(b)

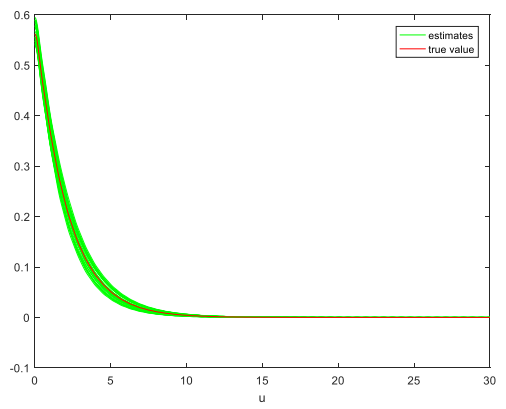

(c)

Figure 3. Estimation of the ruin probability for Gamma $(1.5,1.5)$ claim size density: $(\mathbf{a}) T=120$, (b) $T=180$, (c) $T=360$.

Next, based on the same 300 repeated experiments, Figure 4 shows the performance of the average estimation of the corresponding ruin probability under three kinds of claim distribution assumptions for different $T$, and compares it with the truth curve. It can be seen that with the increase of parameter $T$, the average estimated value is more and more close to the real value, and gradually coincides with the real value, which is difficult to distinguish.

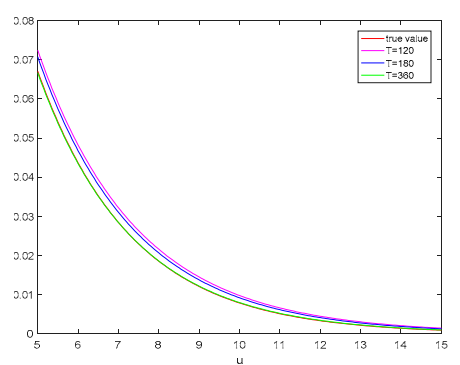

(a)

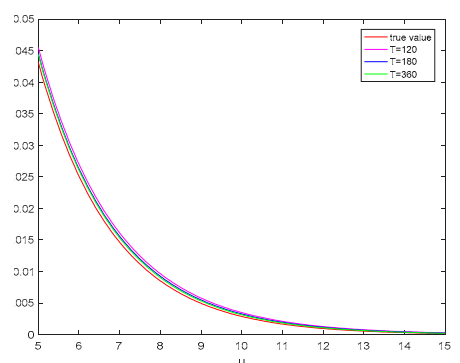

(b)

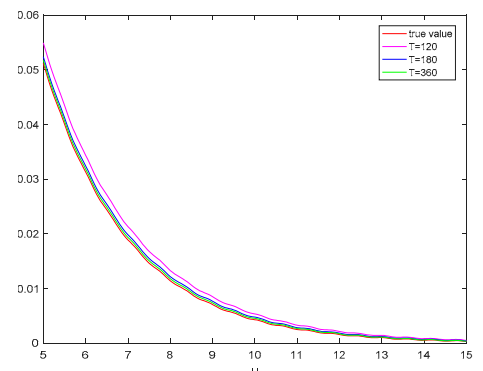

(c)

Figure 4. Estimation of the ruin probability: mean curves. (a) Exponential claim size density; (b) Erlang (2) claim size density; (c) Gamma $(1.5,1.5)$ claim size density. 
To further illustrate the accuracy of the proposed method, we show the average relative error curves under three claims distribution assumptions in Figure 5. Once again, we find that with the increase of observation time $T$, the average relative error decreases, which also means that when $T$ increases, our estimation method performs well and becomes more accurate. Of course, Figure $5 c$ is different from the relative error curve of the other two distributions. The main reason is that the reference truth value used in this paper under the assumption of this distribution is our CFS approximation. More rigorous calculation method of truth value needs further study. But from this Figure, we find that when the initial surplus is in the range of $15-25$, the average relative error fluctuates greatly. The corresponding average estimated value curve is proposed separately, as shown in Figure 6. It is found that in this interval, the estimated value begins to fluctuate, and there is a smaller approximation with the reference true value, which makes the performance of the corresponding relative error curve reasonable.

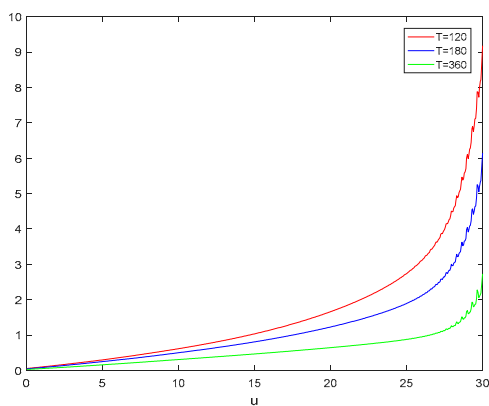

(a)

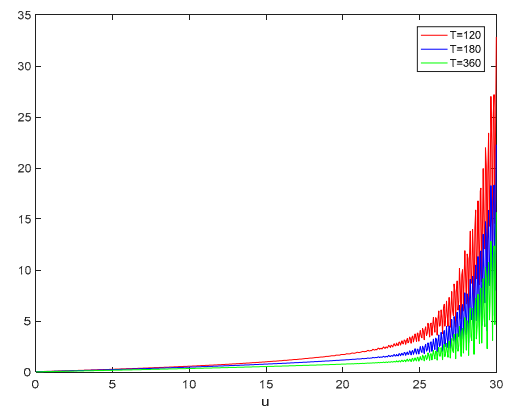

(b)

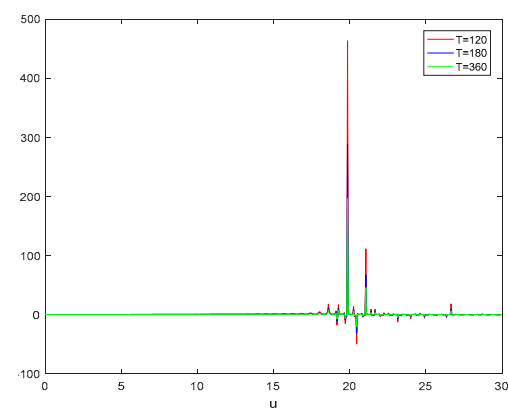

(c)

Figure 5. Estimation of the ruin probability: mean relative error curves. (a) Exponential claim size density; (b) Erlang (2) claim size density; (c) Gamma $(1.5,1.5)$ claim size density.

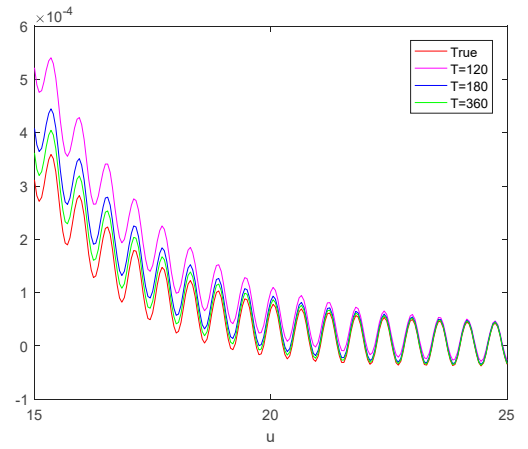

Figure 6. A partial enlargement of the mean curves for the estimation of the ruin probability under the Gamma $(1.5,1.5)$ distribution.

Finally, based on the above 300 repeated experiments, we give a series of IMSE values of ruin probability estimation under three kinds of claim distribution assumptions in Table 1. For each claim distribution, we find that IMSE decreases with the increase of $T$, which is consistent with our conclusion. This conclusion shows the stability of the estimation method in this paper, gives us a reference to improve the accuracy of data collection and provides the necessary basis for deeper application level analysis.

Table 1. IMSEs of ruin probability estimation for different claim distributions and $T$.

\begin{tabular}{clll}
\hline & $T=120$ & $T=180$ & $T=360$ \\
\hline Exponential density & 0.0097 & 0.0062 & 0.0026 \\
Erlang (2) density & 0.0052 & 0.0039 & 0.0021 \\
Gamma (1.5, 1.5) density & 0.0069 & 0.0042 & 0.0023 \\
\hline
\end{tabular}


All numerical experiments in this chapter are based on MATLAB software. Taking the exponential density as an example, when $T=120$, we completed 300 independent repeated experiments in $68.096740 \mathrm{~s}$.

\section{Conclusions}

This paper introduces how to use the CFS method to approximate the ruin probability under the stochastic premium income insurance risk model and gives a nonparametric estimation of the corresponding ruin probability. First, we approximate the ruin probability under the model according to the principle and method of the CFS given. Then, using the sample data set of the number of claims and the size of a single claim on the observation interval, the data set consisting of the number of random premium income and the amount of insurance premiums, a non-parametric estimation of the ruin probability is constructed. Finally, we verify the effectiveness of the method in this paper through error analysis and numerical experiments. The results show that even if it is extended to a more complex model, as long as the Fourier transform of the specific claim distribution under the corresponding model can be obtained, the method can be effectively applied. The conclusion that the numerical simulation is extremely stable not only further demonstrates the superiority of the estimation method in this paper, but also provides a necessary reference for deeper application research.

Author Contributions: Methodology, Y.H. and J.L.; Software, J.L. and H.L.; Writing—original draft preparation, Y.H., J.L. and W.Y. All authors have read and agreed to the published version of the manuscript.

Funding: This research was supported by the Key Projects of National Social Science Foundation of China(No. 21AZD071), the National Natural Science Foundation of China (No. 11301303), the Taishan Scholars Program of Shandong Province (No. tsqn20161041), the Humanities and Social Sciences Project of the Ministry Education of China (No. 19YJA910002), the Natural Science Foundation of Shandong Province (No. ZR2018MG002), the Fostering Project of Dominant Discipline and Talent Team of Shandong Province Higher Education Institutions (No. 1716009), the Shandong Provincial Social Science Project Planning Research Project(No. 19CQXJ08), the 1251 Talent Cultivation Project of Shandong Jiaotong University, the Risk Management and Insurance Research Team of Shandong University of Finance and Economics, the Shandong Jiaotong University 'Climbing' Research Innovation Team Program, and the Collaborative Innovation Center Project of the Transformation of New and Old Kinetic Energy and Government Financial Allocation, Excellent Talents Project of Shandong University of Finance and Economics.

Institutional Review Board Statement: Not applicable.

Informed Consent Statement: Not applicable.

Data Availability Statement: Not applicable.

Conflicts of Interest: The authors declare that they have no conflict of interest.

\section{References}

1. Albrecher, H.; Constantinescu, C.; Loisel, S. Explicit ruin formulas for models with dependence among risks. Insur. Math. Econ. 2011, 48, 265-270. [CrossRef]

2. Mitric, I.; Badescu, A.L.; Stanford, D.A. On the absolute ruin problem in a Sparre Andersen risk model with constant interest. Insur. Math. Econ. 2012, 50, 167-178. [CrossRef]

3. Li, S.M.; Sendova, K.P. The finite-time ruin probability under the compound binomial risk model. Eur. Actuar. J. 2013, 3, $249-271$. [CrossRef]

4. Bayraktar, E.; Zhang, Y.C. Minimizing the probability of life time ruin under ambiguity aversion. SIAM J. Control Optim. 2015, 53, 58-90. [CrossRef]

5. Li, J.C.; Dickson, D.C.M.; Li, S.M. Some ruin problems for the MAP risk model. Insur. Math. Econ. 2015, 65, 1-8. [CrossRef]

6. Mitric, I.R.; Trufin, J. On a risk measure inspired from the ruin probability and the expected deficit at ruin. Scand. Actuar. J. 2016, 2016, 932-951. [CrossRef]

7. Li, J.Z. A note on the finite-time ruin probability of a renewal risk model with Brownian perturbation. Stat. Probabil. Lett. 2017, 127, 49-55. [CrossRef] 
8. Han, X.; Liang, Z.B.; Yuen, K.C. Minimizing the probability of absolute ruin under the mean-variance premium principle. Optim. Contr. Appl. Met. 2021. [CrossRef]

9. Zhang, Z.M.; Yang, H.L.; Yang, H. On a nonparametric estimator for ruin probability in the classical risk model. Scand. Actuar. J. 2014, 4, 309-338. [CrossRef]

10. Chau, K.W.; Yam, S.C.P.; Yang, H.L. Fourier-cosine method for ruin probabilities. J. Comput. Appl. Math. 2015, 281, 94-106. [CrossRef]

11. Goffard, P.O.; Loisel, S.; Pommeret, D. A polynomial expansion to approximate the ultimate ruin probability in the compound Poisson ruin model. J. Comput. Appl. Math. 2016, 296, 499-511. [CrossRef]

12. Yang, Y.; Su, W.; Zhang, Z.M. Estimating the discounted density of the deficit at ruin by Fourier cosine series expansion Stat. Probabil. Lett. 2019, 146, 147-155. [CrossRef]

13. Wang, Y.Y.; Yu, W.G.; Huang, Y.J. Estimating the Gerber-Shiu function in a compound Poisson risk model with stochastic premium income. Discrete Dyn. Nat. Soc. 2019, 2019, 1-18. [CrossRef]

14. Wang, Y.Y.; Yu, W.G.; Huang, Y.J.; Yu, X.L.; Fan, H.L. Estimating the expected discounted penalty function in a compound Poisson insurance risk model with mixed premium income. Mathematics 2019, 7, 305. [CrossRef]

15. Huang, Y.J.; Yu, W.G.; Pan, Y.; Cui, C.R. Estimating the Gerber-Shiu expected discounted penalty function for Lévy risk model. Discrete Dyn. Nat. Soc. 2019, 2019, 1-15. [CrossRef]

16. Yu, W.G.; Yong, Y.D.; Guan, G.F.; Huang, Y.J.; Su, W.; Cui, C.R. Valuing guaranteed minimum death benefits by cosine series expansion. Mathematics 2019, 7, 835. [CrossRef]

17. Zhang, Z.M.; Yong, Y.D.; Yu, W.G. Valuing equity-linked death benefits in general exponential Lévy models. J. Comput. Appl. Math. 2020. [CrossRef]

18. Su, W.; Yu, W.G. Asymptotically normal estimators of the Gerber-Shiu function in classical insurance risk model. Mathematics 2020, 8, 1638. [CrossRef]

19. Ai, M.Q.; Zhang, Z.M.; Yu, W.G. First passage problems of refracted jump diffusion processes and their applications in valuing equity-linked death benefits. J. Ind. Manag. Optim. 2021. [CrossRef]

20. Wang, Y.Y.; Zhang, Z.M.; Yu, W.G. Pricing equity-linked death benefits by complex Fourier series expansion in a regime-switching jump diffusion model. Appl. Math. Comput. 2021. [CrossRef]

21. Cheung, E.C.K.; Zhang, Z.M. Simple approximation for the ruin probability in renewal risk model under interest force via Laguerre series expansion. Scand. Actuar. J. 2021. [CrossRef]

22. Chan, T.L.R. An orthogonal series expansion method to hedge and price European-type options. SSRN 2016. [CrossRef]

23. Chan, T.L.R. Hedging and pricing early-exercise options with complex Fourier series expansion. N. Am. J. Econ. Financ. 2020. [CrossRef]

24. Li, J.; Yu, W.G.; Liu, C.L. Nonparametric estimation of ruin probability by complex Fourier series expansion in the compound Poisson model. Commun. Stat. Theor. Methods 2020. [CrossRef]

25. Zhang, Z.M. Nonparametric estimation of the finite time ruin probability in the classical risk model. Scand. Actuar. J. 2017, 5, 1-18. [CrossRef] 